Available online at http://docs.lib.purdue.edu/jate

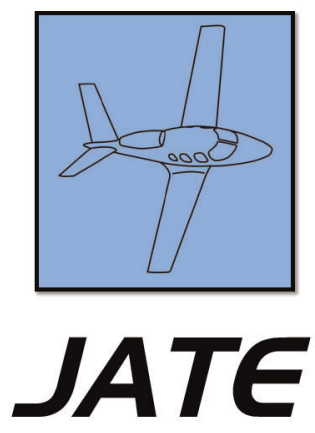

Journal of Aviation Technology and Engineering 10:1 (2021) 2-11

\title{
Design of Rotorcraft Performance-Based Navigation Routes and Procedures: Current Challenges and Prospects
}

\author{
Carlos Gonzaga-Lopez
}

Austro Control GmbH

\begin{abstract}
Helicopters play a relevant role in society due to their extraordinary versatility. However, they are particularly vulnerable to adverse weather conditions as the majority of operations are carried out under visual flight rules. This is partly due to the shortage of tailored helicopter instrument flight procedures and routes. The emergence of the performance-based navigation concept supported by the latest satellite navigation technologies has opened up new possibilities for rotorcraft operations in the last few years. This paper presents an extensive overview of the state of the art in the design of performance-based navigation routes for helicopters from two main standpoints: instrument flight procedures and route spacing. Apart from summarizing recent and current major initiatives to implement helicopter lowlevel routes and flight procedures, this paper provides an outlook on the latest advances and ongoing efforts by the International Civil Aviation Organization in the field of helicopter procedure and route design to ensure flyability, obstacle clearance, strategical separation, and segregation of traffic flows. In addition, several gaps in the current design criteria are identified and suggestions for future research and development are outlined.
\end{abstract}

Keywords: GNSS, performance-based navigation, low-level routes, rotorcraft, route spacing, flight procedures

\section{Introduction}

\subsection{Instrument Flight Rules in the Rotorcraft Domain}

Due to their excellent maneuverability and capability to perform vertical take-off and landing, helicopters play an important role in challenging environments where fixed-wing aircraft have proved insufficient. This holds true not only in the field of regular passenger transport and air freighting, but especially in the execution of critical missions such as aerial firefighting, helicopter emergency medical services (HEMS), search and rescue, and law enforcement among others. In the near future, it is expected that new types of rotorcraft, such as the first civil tilt-rotor (Leonardo, 2020) and multirotor aircraft (ADAC Luftrettung, 2020), can complement the role of helicopters in the provision of these indispensable services for society (Chappelle et al., 2018). 
Most rotorcraft operations are conducted at low altitude and in visual meteorological conditions (VMC). The urge to complete mission-critical operations during unstable weather conditions usually leads to inadvertent instrument meteorological conditions (IMC) encounters at a certain point of the flight. Inadvertent flight into IMC and Controlled Flight Into Terrain-mainly to avoid inclement weather or cloud ceilings-are considered to be some of the main causes of fatal helicopter accidents (U.S. Joint Helicopter Safety Analysis Team, 2011, 2017), particularly in the HEMS business (Blumen, 2002; Rigsby, 2005). In the light of a statistical study on HEMS analyzing crash reports in the United States dating from 1983 to 2005, it can be stated that the majority of crashes with fatalities are associated with missions in darkness or bad weather (Baker et al., 2006). The fact that nighttime accident rates are significantly greater than those of the daytime and that they are mostly attributable to operational issues are confirmed by Nascimento et al. (2014). Instrument flight rules (IFR) capabilities along with crew experience are considered to be an efficient mitigation to reduce the high rate of accidents in the helicopter sector (Blumen, 2002). Visual flight rules (VFR) is the standard way to conduct HEMS missions if weather conditions are appropriate because it normally offers more flexibility and lower workload. Nevertheless, as soon as weather conditions deteriorate, some VFR HEMS flights must be cancelled or cannot be completed for safety reasons, preventing people from receiving immediate medical care. Consequently, many HEMS operators consider investing in IFR capabilities to increase safety and accessibility in bad weather conditions. However, for some operators, the current shortage of specific IFR flight procedures and routes adapted to the nature of rotorcraft operations does not make up for the complex and expensive IFR certification and approval processes. IFR rotorcraft operations require flight procedures tailored to the needs of rotary-wing aircraft and to the particularities of these types of operations. In general terms, procedural altitudes should be as low as possible to avoid icing conditions. Lower flight altitudes can also help minimize the impact of reduced atmospheric pressure on critical patients given the fact that nowadays practically every medical transport by helicopter is carried out with non-pressurized cabins.

\subsection{Performance-Based Navigation for Rotorcraft Operations}

The introduction of the performance-based navigation (PBN) (International Civil Aviation Organization [ICAO], 2012) concept in 2008 marked a milestone in the aviation sector worldwide. This concept represents a paradigm shift from equipment- or sensor-based to performance-based navigation solutions and is key to accommodating the future growth of civil aviation. PBN is based on area navigation (RNAV), which is a mode of navigation that enables flight operations on any desired flight path within the area of coverage of the corresponding navigation infrastructure. RNAV allows for a more efficient use of airspace since it eliminates the need to follow tracks defined by a sequence of conventional ground-based navigation aids to fly from point $\mathrm{A}$ to point $\mathrm{B}$.

One of the main pillars of $\mathrm{PBN}$ is the navigation specification (ICAO, 2012). Apart from a set of functional and aircrew requirements, the navigation specification contains performance requirements that the aircraft must be able to fulfill. The performance levels required for a given navigation specification may be achieved by using different navigation sensors or combining them, e.g., VHF omnidirectional radio range combined with distance measuring equipment or stand-alone satellite navigation systems. The PBN specification can be RNAV or RNP (required navigation performance). Only RNP navigation specifications require on-board performance monitoring and alerting. A summary of the current PBN specifications is shown in Table 1. The number included in most of the navigation specification denotes the maximum total system error in nautical miles (95\% of flight time). Navigation specifications that do not include this number in their designation (i.e., A-RNP, RNP APCH, and RNP AR $\mathrm{RPCH})$ have a variable maximum total system error depending on the phase of flight (see Table 1). It is important to note that currently, RNP monitoring and alerting requirements can only be achieved by using global navigation satellite systems (GNSS).

The latest developments in the field of GNSS have enabled aircraft to fly more safely and more accurately in an RNAV context without the need of ground-based navigation aids. GNSS RNAV routes and flight procedures are significantly contributing to capacity and accessibility improvements all over the world while reducing the environmental impact of flight operations. While these routes have been extensively developed for fixed-wing aircraft during the last years, they are still very limited for rotary-wing aircraft.

The navigation specification RNP 0.3 (Federal Aviation Administration [FAA], 2016a; ICAO, 2012) was conceived in the early 2000s out of the need to enable helicopter operators flying IFR to have a real benefit from PBN. This is not always possible with other navigation specifications, such as RNP 1, considering the challenging environment in which helicopters operate (see Figure 1). RNP 0.3 has become the standard for advanced helicopter procedures throughout all phases of flight except in final, where RNP APCH with linear or angular guidance sensibility applies (see Table 1).

ICAO first recommended the development of RNAV procedures supported by GNSS for both fixed- and rotarywing aircraft during its eleventh Air Navigation Conference in 2003 (ICAO, 2010a). The helicopter community has 
Table 1

Navigation specification and associated maximum total system error per flight phase (based on ICAO, 2020)

\begin{tabular}{|c|c|c|c|c|c|c|c|c|}
\hline & \multicolumn{8}{|c|}{ Flight phase } \\
\hline & \multirow[b]{2}{*}{ Oceanic or remote } & \multirow[b]{2}{*}{ En-route } & \multirow[b]{2}{*}{ Arrival } & \multicolumn{4}{|c|}{ Approach } & \multirow[b]{2}{*}{ Departure } \\
\hline & & & & Initial & Intermediate & Final & Missed & \\
\hline RNAV 10 & 10 & & & & & & & \\
\hline RNAV 5 & & 5 & 5 & & & & & \\
\hline RNP 4 & 4 & & & & & & & \\
\hline RNP 2 & 2 & 2 & & & & & & \\
\hline RNAV 2 & & 2 & 2 & & & & & 2 \\
\hline A-RNP & 2 & 2 or 1 & $1-0.3$ & $1-0.3$ & $1-0.3$ & 0.3 & $1-0.3$ & $1-0.3$ \\
\hline RNP 1 & & & 1 & 1 & 1 & & 1 & 1 \\
\hline RNAV 1 & & 1 & 1 & 1 & 1 & & 1 & 1 \\
\hline RNP 0.3 (Cat H) & & 0.3 & 0.3 & 0.3 & 0.3 & & 0.3 & 0.3 \\
\hline RNP APCH & & & & 1 & 1 & 0.3 & 1 & \\
\hline $\begin{array}{l}\text { RNP APCH } \\
\text { (LP/LPV only) }\end{array}$ & & & & 1 & 1 & Angular & $\begin{array}{c}1 \text { or } 0.3 \text { (initial straight } \\
\text { missed app.) }\end{array}$ & \\
\hline RNP AR APCH & & & & $1-0.1$ & $1-0.1$ & $0.3-0.1$ & $1-0.1$ & \\
\hline
\end{tabular}

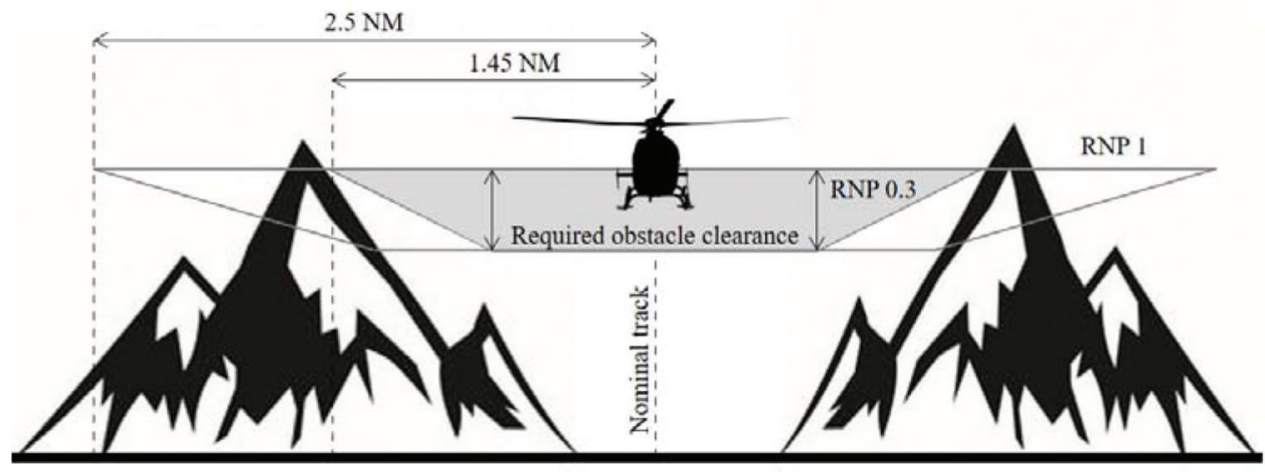

Figure 1. Comparison of obstacle protection areas for rotorcraft routes (RNP 1 versus RNP 0.3).

already begun to draw on this technology to overcome weather limitations. The work carried out by ICAO and its member states in the fields of aircraft operations, flight procedure design, and air traffic management during the last few decades has contributed to the implementation of the first PBN rotorcraft operations. Advanced GNSS procedures to and from a point in space (PinS) in the vicinity of a helicopter landing site (ICAO, 2020) in combination with low-level routes (LLR) (Single European Sky ATM Research Joint Undertaking [SESAR], 2016) have now the potential to better leverage IFR for rotorcraft.

\subsection{Recent Rotorcraft PBN Implementations and Trials}

Several networks of low-level helicopter PBN routes connecting hospitals have already been implemented in different regions of the contiguous United States. The Northeast network, known as the North East Air Alliance, is a good example of a cooperative undertaking where several HEMS and air traffic service providers share information and resources to optimize operations. The first operational HEMS missions in the area were conducted in
2015. These routes and procedures are usually private and are therefore not published in the Aeronautical Information Publication. In 2017, the Radio Technical Commission for Aeronautics (RTCA) Tactical Operations Committee approved a new set of recommendations towards the implementation of an efficient and resilient PBN-centric National Airspace System (NAS) in the United States (RTCA, 2017). The recommendations encourage future publication of as many helicopter routes and procedures as possible to increase operator use and reduce maintenance costs, which would be borne by the FAA. In this respect, the recommendations reflect the need to incorporate IFR rotorcraft operations into the low-altitude airspace supported by an extensive deployment of dedicated PBN public routes (TK-routes) separated from fixed-wing traffic. As of today, only two public TK-routes based on the navigation specification RNAV-2 (FAA, 2016a) have been published between the metropolitan areas of Washington, DC, and New York (FAA, 2011). As per RTCA (2017), feeder routes or transitions providing an IFR connection through PinS procedures between helicopter landing sites and the NAS should also be provided. Furthermore, the 
recommendations consider the introduction of "let-down" or "cloud-break" waypoints along the routes with lower minimum altitudes to facilitate IFR cancellation and subsequent VFR operation. A demonstration project pushed forward by the FAA together with the Maryland State Police and the Potomac Terminal Radar Approach Control was launched in 2017 to study the feasibility of rotorcraft routes and PinS procedures based on RNP 0.3 and the use of constant-radius turns. Some of the procedures in the project were successfully test-flown in 2018 and further validations are underway.

In the remote area of Alaska, most operators are GPSequipped but the benefit is still limited as no proper PBN route network has yet been implemented. A major modernization of the Alaskan NAS combining point-topoint operations with lower minimum altitudes and PBN LLRs has been envisaged in RTCA (2017). PBN routes with highly demanding navigation performances are indispensable in the remote territories of Alaska due to the absence of surveillance in a very demanding mountainous environment.

Unlike Alaska, the operational concept developed in the Gulf of Mexico does not require PBN LLRs to enable safe offshore flight operations to and from the many oil and natural gas platforms in the Gulf. Instead, the routing system is based on an extensive GPS grid of waypoints. By means of a number of different special instrument operations defined in FAA (2017), operators flying under IFR can reach a protected area along the route from which a safe letdown can be executed. The crew can then proceed to land if the required visual conditions are met. Otherwise, the missed approach is to be executed. The use of a weather radar in ground mapping mode is essential to provide the flight crew with a picture of potential obstructions over sea level. These instrument operations require specific approval by the FAA (FAA, 2017). The operational efficiency in the area increased substantially after the introduction of reduced separation minima based on Automatic Dependent Surveillance Broadcast and VHF communication in 2009.

Even though the implementation of PinS procedures combined with LLR networks is still in a very incipient stage in Europe, the benefits of these solutions have already been proved through several EU-funded research and development projects as well as through validation activities such as GIANT, GARDEN, PROUD, HEDGE, 5-LIVES, and OPTIMAL among others. Some other projects, such as SESAR 2020 General Aviation Improved Navigation and Surveillance, are currently being conducted to demonstrate that more affordable avionics for general aviation, including helicopters, are also suitable for advanced navigation applications, such as constant-radius turn capability. These demonstrations are key for the future development of IFR rotorcraft operations and suggest that there is still considerable potential in the latest cutting-edge navigation solutions that is not being exploited to increase safety and accessibility in poor weather conditions.

Although some countries, such as Italy, have already implemented basic helicopter IFR routes, Norway and Switzerland have clearly taken the lead in the implementation of these routes in the European continent. Two main helicopter routes based on RNP 0.3 and Satellite-based Augmentation System (SBAS) are already being used in Switzerland for military and HEMS operations. One of the routes crosses the country from northeast to southwest while the other does it from north to south over very demanding alpine terrain. A second implementation phase will bring additional routes all over the country to connect main hospitals, operator bases, and some aerodromes. The whole route network is being designed to operate in controlled airspace. In the recent past, the Swiss Air Navigation Services Provider (ANSP) together with the main HEMS operator among other international partners have played a pivotal role in the design and demonstration of the first helicopter procedures with an RNP as demanding as $0.1 \mathrm{NM}$ and constant-radius turns in the framework of projects such as PROUD and 5-LIVES. Moreover, Switzerland is currently participating in further research projects to develop advanced procedure design criteria for helicopters with high-performance navigation capabilities to enable IFR operation in extremely complex terrain. In Norway, a massive LLR network based on RNP 1 with up to 17 routes is already available for authorized operators. Most of the helicopter routes in Norway are located in uncontrolled airspace. In addition to the routes, RNP 0.3 transitions have also been developed to connect the LLR network with proprietary PinS departures and approaches. Apart from Italy, Norway, and Switzerland, many other states in Europe will soon implement their first helicopter LLRs while the number of PinS procedures in operation keeps growing steadily.

In the end, an extensive and harmonized use of IFR rotorcraft routes and flight procedures will only become commonplace if the main issues and challenges can be overcome. Current challenges range from areas such as regulations, training, and human factors to other aspects of a more technical nature such as the provision of weather information and airspace design. This paper focuses only on the airspace design discipline and more specifically on flight procedure and route spacing, describing the status quo and way forward.

\section{Instrument Flight Procedure Design and Route Spacing}

An instrument flight procedure is a description of a series of predetermined maneuvers by reference to flight instruments (ICAO, 2009). For some regulators and organizations only terminal procedures such as standard instrument arrivals, instrument approach procedures, and standard 
instrument departures are instrument flight procedures even though en-route criteria are also covered in the procedure design standards.

Flight procedure and airspace designers ensure that the procedures and routes are adequately protected from obstacles and consider other aspects such as automation, communications, navigation, and surveillance technologies, pilot workload, and environmental impact in the design.

A good airspace design also provides enough strategic separation to aircraft on adjacent instrument flight procedures or routes, thus reducing the need for tactical air traffic control (ATC) deconfliction while increasing airspace capacity and efficiency. This is known as route spacing and it is broadly related to the concept of lateral aircraft-toaircraft separation (ICAO, 2016).

These two areas are essential-although not enough on their own-to implement safe and efficient flight operations.

\subsection{Current Standards for Rotorcraft}

\subsubsection{Flight procedure design}

ICAO PANS-OPS (ICAO, 2020) defines standard criteria to design flyable instrument flight procedures with the required protection from obstacles. Although these design criteria were initially developed for fixed-wing aircraft, the particularities of rotorcraft operation have slowly made their way into the ICAO provisions. Helicopter operations were firstly incorporated into ICAO PANS-OPS in 1982 in a very limited fashion. In 1990 the first flight procedures specifically conceived for helicopters only were included into PANS-OPS. With the advent of the first RNAV procedures supported by GNSS in the late 1990s, all design criteria applicable to helicopters were revisited and integrated across the document. Basically, parameters such as the maximum aircraft dimension, the distance to the start of climb after a go-around, characteristic speeds, vertical gradients, height over runway threshold, and minimum turn altitude for departures were specifically established for helicopters. These parameters led to reduced required obstacle clearances, shorter minimum segment lengths, and narrower protection areas applicable to helicopter IFR flight procedures. But it was not until 2004 that the first basic criteria for GNSS-based RNAV PinS procedures saw the light. This milestone enabled access to VFR helicopter landing sites through specific IFR procedures. PinS procedures comprise both an instrument and a visual segment. For approach procedures, the instrument segment is aligned with a reference point (PinS) from which the pilot can maneuver to land in adequate visual conditions or initiate a missed approach if the visual conditions are insufficient. For a PinS departure, the procedure works the other way around. In this case, the visual segment precedes the instrument segment, which begins at the PinS. PinS procedures allow for minimums as low as $250 \mathrm{ft}$ above the elevation of the landing location depending on the obstacle environment.

PinS design criteria have been reviewed several times since their first introduction and are still being refined by the ICAO Instrument Flight Procedure Panel (IFPP). The last PANS-OPS amendment (ICAO, 2020), in effect as of November 2021, includes the possibility of an early entry into IMC during the PinS departure while still on the direct visual segment. This is only foreseen for "proceed visually" PinS departures (without VFR segment) through the fulfillment of some additional requirements to maintain safety levels. Helicopters flying a PinS departure with a "proceed VFR" segment are not allowed to enter IMC prior to the PinS since the obstacle protection begins at that very point. Another novelty, which will offer more flexibility to procedure designers and operators, is the application of a reduced obstacle clearance based on the height-loss ${ }^{1}$ concept to protect the visual transition area of SBAS localizer performance with vertical guidance (LPV) approaches ending with a "proceed visually" segment. LPV approaches are very similar to the conventional ILS approaches but offer more stability and flexibility in general terms.

\subsubsection{Route spacing}

The significant work on separation minima and route spacing developed during the last few years by the ICAO Separation and Airspace Safety Panel (SASP) has led to many of the provisions contained in PANS-ATM (ICAO, 2016). PANS-ATM requirements and procedures are used worldwide to provide air traffic services, including ATC.

As per PANS-ATM, there are two different options to establish the minimum track separation between two nonintersecting segments in the terminal area. The first one is determining the minimum distance for which the corresponding obstacle protection areas (ICAO, 2020) do not overlap. In this case, the operational error has to be taken into account by, for example, adding a given separation buffer between protection areas to achieve the required TLS of $5 \times 10^{-10}$ collisions per operation (aircraft pair). The steps for deriving the separation buffer can be found in ICAO (2010b). The separation buffer can be replaced by other risk-mitigation measures (e.g., ATC monitoring). The second method to establish the minimum spacing is based on the direct application of a collision risk model (CRM) using multiple navigation specifications. For a combination between RNAV 1 and any other PBN specification equal to or better than RNAV 1 (including RNP specifications), the resulting minimum distance between segments yields $7 \mathrm{NM}$. If the combination is only between RNP specifications, the resulting minimum distance yields $5 \mathrm{NM}$. More information on the derivation of these distances using the CRM can be found in ICAO (2010b). As described by

\footnotetext{
${ }^{1}$ Aircraft height loss between the initiation of the go-around maneuver and the point of lowest descent.
} 
De Smedt et al. (2017), these values could be further reduced through the collection and analysis of current data sets. This study demonstrated that the actual navigation performance at some major hubs in Europe is much better than that assumed for the calculation of the current ICAO route separation minima (ICAO, 2010b). By computing the risk of collision for various route configurations, it was found out that the resulting minimum route spacing is more limited by the surveillance separation minima (3 NM in terminal airspace) and a number of human factors, rather than by the navigation performance.

An alternative method based on the risk of loss of separation has also been successfully used by the UK Civil Aviation Authority and the British ANSP to assess the minimum route spacing assuming RNAV 1 routes in a monitored terminal area (UK Civil Aviation Authority, 2016). The outcome of this study also suggests values below current ICAO minima (ICAO, 2010b). No specific criteria or guidance material for helicopter route spacing have been developed to date.

\subsection{Ongoing and Future Developments for Rotorcraft}

\subsubsection{Flight procedure design}

One of the main operational hurdles identified in the current set of criteria is the width of the protection areas corresponding to the navigation specification RNP 0.3 . Both ICAO PANS-OPS (ICAO, 2020) and the U.S. Standard for Terminal Instrument Procedures (FAA, 2016b) contain RNP 0.3 criteria, although to date PANSOPS provisions are limited to tabulated values of navigational tolerances and protection area widths per phase of flight. As per PANS-OPS, all PBN applications, including RNP 0.3, and except RNP Authorization Required (AR), require additional buffer values dependent on aircraft characteristics and phase of flight to account for possible blunder error and other phenomena causing potential excursions out of the basic protection area $(99.7 \%$ probability of containment) (ICAO, 2020). The addition of such buffers was deemed necessary to make up for the lack of a statistically significant data set. Sufficient flight data would allow for an accurate characterization of the tails of the lateral displacement distributions. Assuming that all helicopters certified for RNP 0.3 operations will use autopilot and coupled flight guidance system to limit the flight technical error, as required by current regulations and certification standards (European Aviation Safety Agency [EASA], 2019; FAA, 2014; ICAO, 2012), a reduction of the current buffer values may be plausible and is being investigated by the ICAO IFPP. In addition, the classical RNAV fly-by turn protection with large overshoot areas seems to overestimate the probability of excursion during the turn given the capabilities of today's on-board navigation systems. Narrower protection areas for RNP 0.3 helicopter flight procedures will contribute to lower minimum altitudes and facilitate thereby critical operations in environments with complex terrain without exposing flight crews to icing conditions.

The obstacle protection during the go-around maneuver for helicopter ILS look-alike approaches has also proved to be extremely conservative. These criteria are based on a fixed-wing model that can limit the possibilities and flexibility of IFR helicopter operations without a real benefit in terms of safety. For that reason, the ICAO IFPP currently works on a new model to better estimate the extent of protection required during the go-around. Such models also build upon an enormous amount of flight data, which unfortunately are still very scarce because of the very limited number of operations taking place at present and the associated high costs. The outcome of a recent meta-analysis carried out by the FAA (2020) could only confirm correlation between the height loss and the vertical path angle to a certain degree. The analysis of the dependence between the vertical path angle and the distance traversed from the initiation of the go-around maneuver to the point at which the helicopter begins to climb did not reach the statistical significance required to develop a valid model.

Another important limitation of the current set of PANSOPS procedure design criteria concerns steep LPV PinS approaches. A stabilized steep approach is a very effective means to reduce the noise footprint in highly densely populated areas and can also be used to avoid obstacles or restricted airspace. Very few fixed-wing aircraft are certified for steep ILS approaches over $7^{\circ}$ (e.g., DCH-7 and DO-228), $6.3^{\circ}$ being the maximum covered by ICAO PANS-OPS (ICAO, 2020). However, modern helicopters equipped for LPV GNSS approaches are usually certified for steep approaches up to $6^{\circ}$, reaching even $9-10^{\circ}$ in some cases depending on the avionics (e.g., H145 and AW109) (Rollet et al., 2008). The $6.3^{\circ}$ limitation on the vertical path angle is not a physical limitation, but rather an intrinsic limitation of the procedure design tools and methodologies. The protection areas used currently for LPV PinS approaches are practically the same set of sloping planar surfaces that have been used during roughly the last four decades to estimate safe obstacle clearance heights for ILS approaches flown by fixed-wing aircraft. These funnelshaped surfaces are known as ILS obstacle assessment surfaces and were developed to approximate the risk contours of $10^{-7}$ collisions per approach calculated by the ILS CRM software (ICAO, 1980, 2020). Unfortunately, the current obstacle assessment surfaces do not reflect today's navigational capabilities such as GNSS and its augmentation systems since the statistical distributions used by the CRM date from the 1960s.

Some states also require SBAS availability to fly routes based on RNP 0.3. Despite the fact that this is not required according to the ICAO PBN Manual (ICAO, 2012), it has been demonstrated that using SBAS in complex terrain not 
only provides enhanced signal integrity and availability, but also drastically reduces the total system error (Troller et al., 2016). This fact is not yet accounted for in PANSOPS for en-route applications but has potential for the definition of narrower protection areas in the future.

Helicopters can also fly consistent and predictable curved paths by using appropriate on-board avionics capable of executing constant-radius turns. This functionality is key for helicopter LLRs and terminal procedures in obstacle-rich environments and has also proved to be very effective to reduce noise impact. RNAV terminal and approach procedures can be coded with the so-called radius-to-fix (RF) path terminator (Aeronautical Radio, Inc., 2018) to define constant-radius arcs. Protection areas used for RF legs consist of a lateral buffer on each side of the nominal track and do not add the bulky protection for a turn overshoot. Since path terminators cannot be coded for route segments, it would not be possible to use RF legs on a helicopter LLR. Nevertheless, a very similar application known as fixed-radius transition (FRT) was envisaged for fixed-wing aircraft in the en-route phase as an enabler to closer route spacing along turns (ICAO, 2012). In this case, the definition of the arc is slightly different as only one waypoint and a turn radius are coded instead of the three waypoints required for an RF leg (roll-in, roll-out, and center point). The equipment required to fly FRTs can be found in European Organization for Civil Aviation Equipment/RTCA (2014). The current European certification scheme (EASA, 2019) foresees the use of the FRT functionality only in association with RNP 2, RNP 4, and Advanced RNP (A-RNP) specifications. ICAO PANS-OPS does not include yet any FRT criteria as the concept is still under development and additional guidance on its application is needed (ICAO, 2020). This additional guidance may include the use of FRT on helicopter LLRs, not only to increase airspace capacity by reducing the spacing between parallel routes, but also for noise abatement and obstacle avoidance akin to RF legs.

Although the coding of constant-radius curved paths has proved to be one of the most important functionalities in the PBN toolbox, some recent studies have indicated that this concept may pose some problems for RNP values beyond the orders of magnitude that are being considered today for certification (RNP 1 to RNP 0.1). As claimed by Wipf (2018), the constant-radius arcs defined in applications such as RF legs and FRTs do not fulfill the definition of a curved trajectory based on physical laws, thus generating systematic errors. These systematic errors impede the derivation of parametric statistical distributions required to streamline the protection areas used in procedure design without the need of collecting massive flight datasets. Instead of using constant-radius curves, future PBN functionalities demanding predictable curved paths with extremely low RNP values may leverage the physical properties of a specific type of curve to eliminate systematic errors, as expounded by Smerlas et al. (2006). The key property of such curves is that the curvature linearly changes along the arc. These curves are known as clothoid, Euler, or Cornu spirals and are widely used today as track transition curves in the geometric design of railways and roads among many other applications. An innovative method to construct RF trajectories augmented by clothoid segments during roll-in and roll-out maneuvers can be found in Gierszewski et al. (2018).

One of the major limitations with which mission-critical helicopter operators are faced is the accessibility to any possible destination point in adverse weather and in the most expeditious manner possible. With regard to critical missions, the current approach to LLRs lacks flexibility from an operational standpoint. Any deviation from the route via vectoring or direct routing must be cleared by ATC, which is responsible for providing basic obstacle clearance and separation from other traffics in controlled airspace. In a HEMS context, where the time factor is critical and often means the difference between life and death, air ambulance operators seek to reduce the response time as much as possible. In VMC, HEMS crews can fly direct to the pick-up point or scene of injury as they remain responsible for traffic separation and obstacle clearance. In IMC, however, the helicopter would need to follow the corresponding LLR segment in the hope that the destination point can be reached in VMC. In the best-case scenario, the flight crew might be cleared by ATC to proceed direct to a point in the vicinity of the destination point. But even in this case ATC cannot always provide optimal altitude clearances tailored to the mission. Cleared altitudes are usually derived from surveillance minimum altitude or minimum vectoring altitude (MVA) charts (ICAO, 2016), which are widely used by ATC to keep traffics cleared of obstacles and within controlled airspace when diverting them away from defined obstacle-free routes. MVAs are usually too high for rotorcraft and may not suffice to safely transition to the destination point in VMC due to lower cloud ceilings. In case the helicopter needs to fly through uncontrolled airspace to reach the destination point in IMC, the situation worsens as neither surveillance service nor MVAs are guaranteed and the pilot in command remains responsible for avoiding obstacles if no IFR procedure or route is flown. The development of new procedure design criteria for mission-critical helicopter operators to reduce these operational limitations could save many lives in the future. A good start point may be the application or adaptation of existing procedures for offshore operations to an onshore environment. The GNSS grid network in the Gulf of Mexico is used in combination with special helicopter operations such as the so-called helicopter en-route descent areas (HEDAs) (FAA, 2017). HEDAs allow operators to descend from the IFR enroute structure to a radio altitude of $400 \mathrm{ft}$ within a given area cleared of obstructions over the sea. Once VFR 
conditions are reached, the crew can proceed to land. Only one HEDA can serve several helipads. The development of similar criteria for mission-critical onshore operations would require considering the weather factor in a holistic way. Poor-quality weather reports and forecasts can compromise the feasibility of a scheduled flight. In a future scenario where flexible IFR flight procedures are in place to enable expeditious access to the destination point in VMC, more reliable weather nowcast and real-time capabilities may cater for better route planning and lead to higher likelihood of access to the desired point.

Ultimately, the development of procedure design criteria to respond to the rapid proliferation of new navigation technologies and advanced avionic systems is not sufficient if other related areas do not advance together. One of these areas is aeronautical information management and especially the collection of obstacle data. Helicopter routes and terminal procedures require more extensive and reliable obstacle information due to the low procedural altitudes. Current ICAO aeronautical information management provisions for obstacle information collection (ICAO, 2018a, 2018b) do not consider low-level IFR rotorcraft operations. Aware of this situation, some states are working on their own regulations to fill this gap. In lieu of adequate international provisions, these state regulations will ensure that essential obstacle information relevant to PinS procedures and LLRs is properly captured and made available to procedure designers and end users.

\subsubsection{Route spacing}

Taking into consideration that the lowest total system error used in previous risk-based analyses to derive the lateral separation minima for aircraft on published instrument flight procedures was $1 \mathrm{NM}$ (95\% of the flight time), the resulting minima may be excessive in cases where helicopter RNP 0.3 routes are involved. For this reason, the ICAO SASP is working on the development of new lateral separation minima for helicopter operations with RNP 0.3 in line with the ICAO PBN Manual (ICAO, 2012). Values well below the current $5 \mathrm{NM}$ minimum would not be surprising, not only because of the reduced navigational tolerances, but also due to the flight characteristics of rotary-wing aircraft. While this should hold true for a scenario with two parallel helicopter routes, the coexistence of helicopter routes with terminal procedures for fixedwing aircraft in the same terminal area introduces a new term in the equation, namely the variability in the turn performance. As stated in PANS-ATM (ICAO, 2016), any potential separation infringement due to the very nature of the turn shall be mitigated by applying another type of separation or by increasing the applicable separation minima accordingly before the aircraft commences the turn. The probability of violating the prescribed separation minima on parallel routes with fly-by turns can increase considerably in concrete cases if the differences between the turn performance of helicopters and fixed-wing aircraft are not accounted for. Moreover, the lower containment requirements of the RNAV 1 and RNP 1 navigation specifications used by fixed-wing aircraft in terminal areas compared with the state-of-the-art RNP 0.3 navigation specification for rotorcraft in all phases of flight may aggravate the situation and reduce even more the actual spacing at the fly-by turns. It must be stressed that this phenomenon can only be significant if no constant-radius turns are used and both routes share similar vertical profiles. Although closely spaced parallel routes constitute a cutting-edge solution to optimize airspace and increase capacity, shared use by rotorcraft and larger commercial aircraft is not foreseen. This is mainly due to the inherently different lateral tracks required for fixed-wing routes in terminal airspace and helicopter routes. Helicopter lowlevel routes are usually more constrained by the underlying terrain than those developed for fixed-wing aircraft. Even in cases where parallel rotorcraft and fixed-wing routes could be envisaged, the very nature of the different vertical profiles would result in a reduced potential interaction. Further developments in rotorcraft technology (e.g., deicing systems and pressurized cabins) might allow for standard rotorcraft routes at higher altitudes, where the implementation of closely spaced parallel routes (rotorcraft and fixedwing) could help mitigate airspace congestion in the future. This game-changer justifies considering the previously mentioned differences in the turn performance to develop specific route separation minima or additional safety buffers.

It is also worth mentioning that the work carried out by the ICAO SASP to establish safe route spacing minima for RNP 0.3 helicopter operations may also be used as a reference to segregate IFR traffics on terminal procedures and on LLRs within uncontrolled airspace. Not only, but particularly in a scenario with uncontrolled airspace, it must be assumed that in some areas neither surveillance nor communication services may be available. In some other areas, communication services may be possible but without surveillance. The absence of these technological aids limits the capacity to detect conflicts and react accordingly and thus has an important impact on the estimated risk of collision.

\section{Conclusions and Way Forward}

Even though the implementation of IFR helicopter routes and procedures is still scant, there are multiple major initiatives ongoing worldwide to accelerate the paradigm shift that has already been initiated. This paradigm shift is intended to bring the safety benefits of the flights performed under IFR with GNSS support to the rotorcraft community. Areas such as flight procedures and route design are key to enable IFR helicopter operations to gradually become mainstream. In the procedure design 
domain, the existing criteria for the new standard navigation specification RNP 0.3 for helicopters in all phases of flight are still at an early stage. Moreover, this navigation specification may be insufficient in demanding environments with challenging terrain where narrower protection areas and more predictable turn performances through constant-radius turns are required. Nevertheless, the use of constant-radius turns combined with extremely accurate navigation specifications in the future may not automatically lead to narrower protection areas due to the systematic errors inherent to the geometrical construction of these types of curves. In the medium term, the development of new procedure design criteria specific to mission-critical helicopter IFR operations could facilitate the task of reaching the destination point in poor visibility conditions as fast as possible. In the long term, research efforts could focus on sensor-rich autonomous systems capable of calculating the shortest point-to-point flyable path while considering terrain and obstacles in IMC.

Regarding route spacing, the assessment of new separation minima standards for helicopter RNP 0.3 routes is being currently carried out. Reducing the spacing between rotorcraft routes will increase airspace efficiency and capacity in the volumes of airspace in which helicopters regularly operate without increasing the risk of collision. Furthermore, reduced separation minima for rotorcraft could also contribute to the implementation of more efficient simultaneous non-interfering procedures in the future. This would enable independent approach and departure operations of rotorcraft with no ATC interaction and therefore with no disruption of the airliner flow at major hub airports.

The design of rotorcraft routes parallel to standard departure or arrival routes in terminal airspace is practically inconceivable today. However, it is worth considering the differences in the turn performance to analyze lower route spacing if more high-altitude IFR rotorcraft operations can be expected.

In the end, future efforts to progress the fields of procedure design, route spacing, and aircraft-to-aircraft separation must integrate the new developments in communication, navigation, and surveillance techniques and technologies. It is only in this manner that we will be able to cope with the increasing demand for airspace capacity and consolidate the integration of new actors, such as rotary-wing aircraft, into IFR operations.

\section{References}

ADAC Luftrettung. (2020). Multicopter in the rescue service: feasibility study on the application potential of multicopters as emergency doctor shuttles [Result report]. Retrieved from https://press.volocopter. com/images/pdf/ADAC-Luftrettung-Volocopter-feasibility-study2020.pdf

Aeronautical Radio, Inc. (2018). Navigation System Database-ARINC 424-22.
Baker, S. P., Grabowski, J. G., Dodd, R. S., Shanahan, D. F., Lamb, M. W., \& Li, G. H. (2006). EMS helicopter crashes: What influences fatal outcome? Annals of Emergency Medicine, 47(4), 351-356.

Blumen, I. J. (2002). A safety review and risk assessment in air medical transport. Supplement to the Air Medical Physician Handbook. Air Medical Physician Association.

Chappelle, C., Li, C., Vascik, P. D., \& Hansman, R. J. (2018). Opportunities to enhance air emergency medical service scale through new vehicles and operations. In 2018 Aviation Technology, Integration, and Operations Conference. doi: https://10.2514/6.2018-2883

De Smedt, D., Trenevska, D., \& Moek, G. (2017). Using PBN for terminal and extended terminal operations. In Proceedings of the 12th USA/ Europe Air Traffic Management R\&D Seminar (pp. 1-10).

European Aviation Safety Agency. (2019). Certification Specifications and Acceptable Means of Compliance for Airborne Communications, Navigation and Surveillance (CS-ACNS issue 2). Cologne: European Aviation Safety Agency.

European Organization for Civil Aviation Equipment/Radio Technical Commission for Aeronautics. (2014). Minimum Aviation System Performance Standards: Required Navigation Performance for Area Navigation (ED75C/DO236C).

Federal Aviation Administration. (2011). Establishment of Helicopter Area Navigation (RNAV) Routes; Northeast United States, 76 Fed. Reg. 37261 (June 27, 2011). Washington, DC: Federal Aviation Administration.

Federal Aviation Administration. (2014). Airworthiness Approval of Positioning and Navigation Systems (Advisory Circular 20-138D incl. change 2). Washington, DC: Federal Aviation Administration.

Federal Aviation Administration. (2016a). Approval Guidance for RNP Operations and Barometric Vertical Navigation in the U.S. National Airspace System and in Oceanic and Remote Continental Airspace (Advisory Circular 90-105A). Washington, DC: Federal Aviation Administration.

Federal Aviation Administration. (2016b). United States Standard for Performance Based Navigation (PBN) Instrument Procedure Design (Order 8260.58A incl. change 1 and 2). Washington, DC: Federal Aviation Administration.

Federal Aviation Administration. (2017). Approval of Offshore Standard Approach Procedures, Airborne Radar Approaches, and Helicopter En-Route Descent Areas (Advisory Circular 90-80C). Washington, DC: Federal Aviation Administration.

Federal Aviation Administration. (2020). FAA Aviation Safety. Memorandum: Relationship between Along-Track Distance Travelled during Missed Approach and Approach Angle. Washington, DC: Federal Aviation Administration.

Gierszewski, D. M., Schneider, V., Lauffs, P. J., Peter, L., \& Holzapfel, F. (2018). Clothoid-augmented online trajectory generation for radius to fix turns. IFAC-PapersOnLine, 51(9), 174-179.

International Civil Aviation Organization. (1980). Manual on the use of Collision Risk Model (CRM) for ILS operations (Doc 9274), 1 st edition.

International Civil Aviation Organization. (2009). Quality assurance manual for flight procedure design volume 1-Flight procedure design quality assurance system (Doc 9906), 1st edition.

International Civil Aviation Organization. (2010a). ICAO 37th General Assembly Resolution (A37-11): Performance Based Navigation global goals.

International Civil Aviation Organization. (2010b). Circular 324: Guidelines for Lateral Separation of Arriving and Departing Aircraft on Published Adjacent Instrument Flight Procedures.

International Civil Aviation Organization. (2012). Performance-based navigation manual (Doc 9613), 4th edition.

International Civil Aviation Organization. (2016). Procedures for air navigation services-Air traffic management (Doc 4444, PANSATM), 16th edition (incl. Amdt.9). 
International Civil Aviation Organization. (2018a). Annex 15 to the Convention on International Civil Aviation: Aeronautical Information Services, 16th edition.

International Civil Aviation Organization. (2018b). Procedures for air navigation services-Aeronautical information management (Doc 10066, PANS-AIM), 1st edition.

International Civil Aviation Organization. (2020). Procedures for air navigation services volume 2-Construction of visual and instrument flight procedures (Doc 8168, PANS-OPS), 7th edition (including Amdt.9).

Leonardo. (2020). Tokyo Metropolitan Government evaluates the AW609 tiltrotor's unique capabilities to revolutionize connections with Ogasawara island [Press release]. Retrieved from https://www. leonardocompany.com/en/press-release-detail/-/detail/02-09-2020leonardo-tokyo-metropolitan-government-evaluates-the-aw609-tiltrotors-unique-capabilities-to-revolutionize-connections-with-ogasawara-isla? $\mathrm{f}=\% 2$ Fpress-release-detail

Nascimento, F. A., Majumdar, A., \& Ochieng, W. Y. (2014). Helicopter accident analysis. Journal of Navigation, 67(1), 145-161.

Radio Technical Commission for Aeronautics. (2017). Recommendations for the Performance Based Navigation (PBN) Route System.

Rigsby, M. (2005). US civil helicopter emergency medical services (HEMS) accident data analysis, the FAA perspective. In Proceedings of the International Helicopter Safety Symposium (pp. 26-29).

Rollet, P., Authesserre, M., \& Sandri, F. (2008). Flight testing of rotorcraft IFR steep approaches using SBAS and GBAS guidance. In Proceedings of the 64th American Helicopter Society International Annual
Forum, volume 1 (pp. 545-556). American Helicopter Society International.

Single European Sky ATM Research Joint Undertaking. (2016). Solution Guidance 113: Optimised Low-Level IFR routes for rotorcraft (P04.10).

Smerlas, A. J., Stoelzle, M., Melz, C., \& Reich, A. (2006). On the development of a four-axis AFCS for the pilot assistance experimental system. In Proceedings of the 32nd European Rotorcraft Forum (ERF 2006), volume 2 (pp. 960-970).

Troller, M., Scaramuzza, M., Wipf, H., Nyffenegger, M., \& Leibundgut, H. (2016). Flight performance investigations of enhanced rotorcraft operations in mountainous areas-Towards a more ambitious RNP performance. In Proceedings of the 29th International Technical Meeting of Satellite Division of the Institute of Navigation (ION GNSS+ 2016), Portland, Oregon (pp. 3660-3668).

UK Civil Aviation Authority. (2016). CAA Publication (CAP) 1385: Performance-Based Navigation (PBN): Enhanced Route Spacing Guidance, 1st edition.

U.S. Joint Helicopter Safety Analysis Team. (2011). The Compendium Report: The U.S. JHSAT Baseline of Helicopter Analysis. International Helicopter Safety Team, Fairfax, VA.

U.S. Joint Helicopter Safety Analysis Team. (2017). Helicopter Safety Enhancements: Loss of Control-Inflight, Unintended Flight in IMC, and Low-Altitude Operations [Report].

Wipf, H. (2018). Total system error (TSE) analysis of helicopter procedure in an alpine environment flying advanced RNP procedures. In Proceedings of the 20th International Flight Inspection Symposium (IFIS 2018)—ION and ICASC (pp. 195-213). 